\begin{tabular}{|c|l|}
\hline Title & $\begin{array}{l}\text { Mitochondrial-targeted DNA delivery using a DF-MITO-Porter, an innovative nano carrier with cytoplasmic and } \\
\text { mitochondrial fusogenic envelopes }\end{array}$ \\
\hline Author(s) & Y amada, Y uma; Kawamura, Eriko; Harashima, Hidey oshi \\
\hline Citation & $\begin{array}{l}\text { Journal of Nanoparticle Research, 14(8), 1013 } \\
\text { https://doi.org/10.1007/311051-012-1013-3 }\end{array}$ \\
\hline Issue Date & 2012-08 \\
\hline Doc URL & http://hdl.handle.net/2115/50077 \\
\hline Rights & The final publication is available at www.springerlink.com \\
\hline Type & article (author version) \\
\hline File Information & JNR148_1013.pdf \\
\hline
\end{tabular}

Instructions for use 


\section{Mitochondrial-targeted DNA delivery using a DF-MITO-Porter, an innovative nano carrier with cytoplasmic and mitochondrial fusogenic envelopes}

Yuma Yamada $^{\mathrm{a}}$, Eriko Kawamura ${ }^{\mathrm{a}}$, Hideyoshi Harashima ${ }^{\mathrm{a} *}$

${ }^{a}$ Laboratory for molecular design of pharmaceutics, Faculty of Pharmaceutical Sciences, Hokkaido University, Kita-12, Nishi-6, Kita-ku, Sapporo 060-0812, Japan

*Corresponding author: Laboratory for molecular design of pharmaceutics, Faculty of Pharmaceutical Sciences, Hokkaido University, Kita-12, Nishi-6, Kita-ku, Sapporo 060-0812, Japan

Tel: +81-11-706-3919 Fax: +81-11-706-4879

E-mail: harasima@pharm.hokudai.ac.jp 


\begin{abstract}
Mitochondrial gene therapy has the potential to cure a variety of diseases that are associated with mitochondrial DNA mutations and/or defects. To achieve this, it will be necessary to deliver therapeutic agents into the mitochondria in diseased cells. A number of mitochondrial drug delivery systems have been reported to date. However, reports of mitochondrial-targeted DNA delivery are limited. To achieve this, the therapeutic agent must be taken up by the cell (1), after which, the multi-processes associated with intracellular trafficking must be sophisticatedly regulated so as to release the agent from the endosome and deliver it to the cytosol (2) and to pass through the mitochondrial membrane (3). We report herein on the mitochondrial delivery of oligo DNA as a model therapeutic using a Dual Function (DF)-MITO-Porter, an innovative nano carrier designed for mitochondrial delivery. The critical structural elements of the DF-MITO-Porter include mitochondria-fusogenic inner envelopes and endosome-fusogenic outer envelopes, modified with octaarginine which greatly assists in cellular uptake. Inside the cell, the carrier passes through the endosomal and mitochondrial membranes via step-wise membrane fusion. When the oligo DNA was packaged in the DF-MITO-Porter, cellular uptake efficiency was strongly enhanced. Intracellular observation using confocal laser scanning microscopy showed that the DF-MITO-Porter was effectively released from endosomes. Moreover, the findings confirmed that the mitochondrial targeting activity of the DF-MITO-Porter was significantly higher than that of a carrier without outer endosome-fusogenic envelopes. These results support the conclusion that mitochondrial-targeted DNA delivery using a DF-MITO-Porter can be achieved when intracellular trafficking is optimally regulated.
\end{abstract}

Key words: Mitochondria; Mitochondrial-tageted DNA delivery; MITO-Porter; Membrane fusion; Cellular uptake; Endosomal escape. 


\section{Introduction}

To date, a number of investigators have reported that genetic defects in mitochondrial DNA (mtDNA) are associated with mitochondrial dysfunctions, which can lead to the development of a variety of human disorders (Holt et al. 1988; Tuppen et al. 2010; Wallace 2005). It is known that point mutations of mtDNA are factors in the development of mitochondrial diseases, including mitochondrial myopathy, encephalopathy, lactic acidosis and stroke-like episodes (MELAS) (Goto et al. 1990), neurogenic muscle weakness, ataxia, and retinitis pigmentosa (NARP) (Holt et al. 1990) and myoclonic epilepsy with ragged-red fibers (MERRF) (Shoffner et al. 1990). Accordingly, the precise site-specific correction of mtDNA mutations would be expected to be a sophisticated strategy for the treatment of mitochondrial genetic disorders. For this purpose, some approaches using oligo DNA have been investigated in attempts to repair mutated mtDNA and inhibit the replication for mutated mtDNA, although these efforts have been restricted to cell-free systems (Chen et al. 2001; Taylor et al. 1997).

To apply this innovative strategy to mitochondrial gene therapy, in which the mitochondrial genome is the target, it is necessary to deliver therapeutic agents into the innermost mitochondrial space (the mitochondrial matrix), which contains the mtDNA pool. A number of mitochondrial delivery systems for various cargoes have been reported (Mukhopadhyay and Weiner 2007; Yamada and Harashima 2008; Zhang et al. 2011). In the case of low molecular weight drugs, many researchers have reported that mitochondrial targeting activity can be enhanced by chemically conjugating the drugs to molecules that have an affinity for mitochondria (James et al. 2007; Wipf et al. 2005). On the other hand, reports of mitochondrial-targeted DNA delivery are limited. The use of a mitochondrial targeting signal peptide (MTS) makes it possible to selectively deliver linear DNA to isolated mitochondria (Flierl et al. 2003; Seibel et al. 1995; Vestweber and Schatz 1989). However, the MTS is required for the cellular uptake of the cargo, because the MTS conjugate itself cannot be internalized through the cellular membrane. On the other hand, Weissig and coworkers reported on the development of DQAsomes, which are mitochondriotropic and cationic vesicles designed for mitochondrial-targeted DNA delivery (D'Souza et al. 2003; Weissig et al. 2001). They showed that DQAsomes specifically release pDNA proximal to mitochondria in living cells (D'Souza et al. 2003; D'Souza et al. 2005), although the delivery of cargoes into the interior of mitochondria was not confirmed.

To achieve mitochondrial-targeted DNA delivery, multiple processes i.e., (i) cellular uptake, (ii) endosomal escape, (iii) passage through the mitochondrial membrane need to be regulated. In previous studies, we showed that high-density octaarginine (R8)-modified liposomes were internalized primarily via macropinocytosis (Khalil et al. 2006). We also were able to determine the optimal lipid composition for endosome-fusogenic activity to be [1, 2-dioleoyl-snglycero-3-phosphatidyl ethanolamine (DOPE) / phosphatidic acid (PA) / stearyl octaarginine (STR-R8) (7:2:1, molar ratio)], and showed that this optimal lipid composition dramatically enhanced endosomal escape in living cells (El-Sayed et al. 2008; Akita et al. 2009). On the other 
hand, we proposed a MITO-Porter, a liposome-based carrier that is capable of introducing macromolecular cargos into mitochondria via membrane fusion (Yamada et al. 2008). We used fluorescence resonance energy transfer (FRET) analysis to screen liposomes with different lipid compositions for high fusion efficiency using isolated rat liver mitochondria (Yamada et al. 2008). Two highly fusogenic lipid compositions, R8-modified liposomes composed of DOPE and either sphingomyelin (SM) or PA [DOPE/SM/STR-R8 (9:2:1) or DOPE/PA/STR-R8 (9:2:1)], were identified. Moreover, when the lipid composition contained SM, the liposomes had a lower cytotoxicity than that of PA and were judged to be optimal for mitochondrial delivery (Yamada et al. 2011). Furthermore, intracellular observations using confocal laser scanning microscopy (CLSM) and intra-mitochondrial observations by transmission electron microscopy showed that the MITO-Porter successfully delivered macromolecules to mitochondria (Yamada et al. 2008).

We report herein on the mitochondrial delivery of oligo DNA as a model therapeutic agent using the Dual Function (DF)-MITO-Porter, modified with high-density R8 to enhance cellular uptake, incorporating an endosome-fusogenic lipid envelope for endosomal escape and the MITO-Porter for the mitochondrial delivery of a macromolecule (Fig. 1). The critical structural elements of the DF-MITO-Porter include a complexed particle of cargos that are coated with mitochondria-fusogenic inner membranes and endosome-fusogenic outer membranes. Modification of the outer envelope-surface with a high density of R8 greatly assists in the efficient internalization of the carriers into cells (1st step). Inside the cell, the carrier escapes from the endosome into the cytosol via membrane fusion, a process that is mediated by the outer endosomefusogenic lipid membranes (2nd step). The carrier then fuses with the mitochondrial membrane to deliver its cargo into mitochondria (3rd step). Thus, the DF-MITO-Porter appears to be more efficient than conventional MITO-Porter in terms of endosomal escape resulting in a more efficient mitochondrial delivery, when the intracellular trafficking was regulated based on our concept, as shown in Figure 1.

In a previous study, we reported that the DF-MITO-Porter could deliver to mitochondria more efficiently compared to conventional MITO-Porter without the presence of an endosomefusogenic envelope (Yamada et al. 2011). Moreover, we showed that the mitochondrial delivery of DNase I protein by the DF-MITO-Porter drastically decreased mtDNA-levels via mitochondrial specific fusion. However, the intracellular trafficking process of the carriers to their final destination (mitochondria) was unclear. In this study, we evaluated these intracellular multiple processes i.e., (i) cellular uptake, (ii) endosomal escape and (iii) mitochondrial delivery, to assess this issue more carefully. We first carried out flow cytometry analyses to evaluate the cellular uptake efficiency of the carriers. Intracellular observations using CLSM permitted us to compare endosomal escape between the DF-MITO-Porter and a conventional MITO-Porter. Moreover, we obtained the intracellular images after staining mitochondria, and quantified the mitochondrial targeting activity using a confocal image-assisted integrated quantification method. Finally, cell viabilities and mitochondrial membrane potentials were evaluated to exclude cell and mitochondrial toxicities. 


\title{
Experimental
}

\author{
Materials
}

1, 2-dioleoyl-sn-glycero-3-phosphatidyl ethanolamine (DOPE) and 7-nitrobenz-2-oxa-1, 3-diazole labeled DOPE (NBD-DOPE) were purchased from Avanti Polar lipids (Alabaster, AL, USA). Cholesteryl hemisuccinate (5-cholesten-3-ol 3-hemisuccinate; CHEMS), sphingomyelin (SM) and phosphatidic acid (PA) were purchased from Sigma (St. Louis, MO, USA). Stearyl octaarginine (STR-R8) (Futaki et al. 2001) was obtained from KURABO INDUSTRIES LTD (Osaka, Japan). The oligo DNA (5' - CTTCTCGTCCCCATGGATGACCCC-3') and the oligo DNA labeled with fluorescent that Cy5 is conjugated to 5 terminal of the oligo DNA were obtained from Hokkaido System Science Co., Ltd (Sapporo, Japan). HeLa human cervix carcinoma cells were obtained from the RIKEN Cell Bank (Tsukuba, Japan). Dulbecco's modified Eagle medium (DMEM), LysoTracker Blue DND-22, Rhodamine123 and MitoTracker Deep Red 633 were purchased from Invitrogen Corporation (Carlsbad, CA, USA). Fetal bovine serum (FBS) was purchased from Thermo Scientific (Waltham, MA, USA). All other chemicals used were commercially available reagent-grade products.

\section{Preparation of nanoparticle of oligo DNA complexed with STR-R8}

Complexed oligo DNA particles were prepared using STR-R8, which is a good condenser of oligo DNA (Nakamura et al. 2006; Yamada et al. 2005). To prepare an oligo DNA - STR-R8 complex, solutions of oligo DNA $(0.1 \mathrm{mg} / \mathrm{mL})$ were mixed with the STR-R8 solution in $10 \mathrm{mM}$ HEPES buffer (HB, $\mathrm{pH}$ 7.4) under vortexing at a series of nitrogen/phosphate (N/P) ratios.

\section{Construction of a conventional MITO-Porter encapsulating oligo DNA}

A conventional MITO-Porter encapsulating oligo DNA was prepared using the lipid film hydration method as shown in Figure S1. We initially formed nanoparticles of oligo DNA as follows; a solution of oligo DNA $(0.1 \mathrm{mg} / \mathrm{mL})$ was added to the STR-R8 solution under vortexing at an N/P ratio of 3.0. Next, $0.25 \mathrm{~mL}$ of the complexed oligo DNA particle solution was added to the lipid film, which was formed by the evaporation of a chloroform solution of $137.5 \mathrm{nmol}$ lipid [DOPE/SM/CHEMS (9:2:1, molar ratio)], on the bottom of a glass tube, followed by a 15-min incubation to hydrate the lipids. The glass tube was then sonicated for $30 \mathrm{sec}$ in a bath-type sonicator (85 W, Aiwa Co., Tokyo, Japan). A solution of STR-R8 (10 mol\% of lipid) was added to the resulting suspensions to attach $\mathrm{R} 8$ to the surface of the carrier, and the carriers with a mitochondrial fusogenic envelope were used as a conventional MITO-Porter. 
Coating of a conventional MITO-Porter with an endosome fusogenic envelope to produce the DF-MITO-Porter

The Dual Function (DF)-MITO-Porter was constructed by coating the conventional MITO-Porter with a endosome fusogenic envelope by the multi-layering method (Fig. S1), as previously reported (Akita et al. 2009; Yamada et al. 2011). For the outer envelopes, small unilamellar vesicles (SUVs) were prepared by the following procedure. A $1.5 \mathrm{~mL}$ aliquot of $\mathrm{HB}$ was applied to a dried lipid film, formed by evaporation of a chloroform solution of $825 \mathrm{nmol}$ lipid [DOPE/PA ( $7: 2$, molar ratio)], on the bottom of a glass tube. After incubation for 15 min to hydrate the lipids, the suspensions were sonicated using a bath-type sonicator for $30 \mathrm{sec}$. The resulting suspensions were then sonicated using a probe-type sonicator (SONIFIER MODEL 250 D, BRANSON, Danbury, CT, USA) for $10 \mathrm{~min}$ on ice to form negatively charged SUV. The conventional MITOPorter, which is positively charged, was mixed with negatively charged SUV at a ratio of 1:2 (v/v). An STR-R8 solution (10 mol\% of SUV lipid) was then added to the resulting suspension to modify the outer envelope with R8 to produce the DF-MITO-Porter.

\section{Measurement of particle diameter and $\zeta$ potential}

Particle-diameter was measured by a quasi-elastic light scattering method and $\zeta$ potentials were determined electrophoretically by means of an electrophoretic light scattering spectrophotometer (Zetasizer Nano ZS; Malvern Instruments, Herrenberg, Germany).

Quantification of the cellular uptake of the oligo DNA encapsulated carriers by flow cytometry

HeLa cells $\left(2 \times 10^{5}\right.$ cells $)$ were seeded on a 6-well plate (Corning, NY, USA) with DMEM, containing $10 \% \mathrm{FBS}$, under an atmosphere of $5 \% \mathrm{CO}_{2} /$ air at $37^{\circ} \mathrm{C}$ for $24 \pm 3 \mathrm{hr}$. The cells were washed with phosphate-buffered saline (PBS (-)) before incubation with the carriers. The carriers with inner lipid envelopes labeled with 1\% NBD-DOPE or encapsulating oligo DNA labeled with $5 \mathrm{~mol} \%$ of Cy5, were added to the HeLa cells (final inner lipid concentration, $9.2 \mu \mathrm{M}$; final concentration of oligo DNA, $80 \mathrm{nM}$ ). The cells were then incubated in serum-free medium for $1 \mathrm{hr}$ under an atmosphere of $5 \% \mathrm{CO}_{2} /$ air at $37^{\circ} \mathrm{C}$. After removing the medium, the cells were washed once with ice-cold PBS (-) and twice with ice-cold PBS (-) containing heparin $(20 \mathrm{U} / \mathrm{mL})$. The cells were then trypsinized, suspended in DMEM with serum, isolated by centrifugation (700 $g, 4$ ${ }^{\circ} \mathrm{C}, 3 \mathrm{~min}$ ) and washed with FACS buffer (0.5\% bovine serum albumin and $0.1 \% \mathrm{NaN}_{3}$ in PBS (-). After resuspension in $0.5 \mathrm{~mL}$ of FACS buffer, the cell suspension was filtered through a nylon mesh to remove cell aggregates and dust. The cells were then analyzed by flow cytometry (FACScan, Becton Dickinson, Franklin Lakes, NJ, USA). The cells were excited with a $488 \mathrm{~nm}$ light from an Ar laser for detecting NBD and a $635 \mathrm{~nm}$ light from an LD laser for detecting Cy5. 
The two fluorescence detection channels were set to the FL1 filter for NBD and the FL4 filter for Cy5. The cellular uptake of the carriers and oligo DNA were expressed as the mean fluorescence intensity, calculated using the CellQuest software (Becton Dickinson, Franklin Lakes, NJ, USA).

\section{Intracellular observation of oligo DNA encapsulated carriers using CLSM}

HeLa cells ( $4 \times 10^{4}$ cells) were cultured in $35 \mathrm{~mm}$ dishes (BD Falcon, NJ) with DMEM, which contained $10 \% \mathrm{FBS}$, under an atmosphere of $5 \% \mathrm{CO}_{2} /$ air at $37^{\circ} \mathrm{C}$ for $24 \pm 3 \mathrm{hr}$. The DF-MITOPorter or the conventional MITO-Porter encapsulating oligo DNA labeled with $5 \mathrm{~mol} \%$ of Cy5 were added to the HeLa cells (final concentration of oligo DNA, $80 \mathrm{nM}$ ). The cells were then incubated in phenol red-free medium without serum under an atmosphere of $5 \% \mathrm{CO}_{2} /$ air at $37^{\circ} \mathrm{C}$. After a 1-hr incubation, the medium was replaced with fresh phenol red-free medium containing serum, and the cells were incubated in the absence of the carriers for $2 \mathrm{hr}$. The cells were observed by confocal laser scanning microscopy (CLSM) after staining the endosomes or mitochondria.

In the case of endosome staining, 30 minutes before the acquisition of fluorescence images, LysoTracker Blue DND-22 was applied to the medium with serum at a final concentration of $1 \mu \mathrm{M}$. After this incubation, the cells were washed with the phenol red-free medium containing serum, and then observed by CLSM (FV10i-LIV; Olympus Corporation, Tokyo, Japan). The cells were excited with a $405 \mathrm{~nm}$ light for detecting LysoTracker Blue DND-22 and a $635 \mathrm{~nm}$ light for detecting Cy5 from a LD laser. Images were obtained using a FV10i-LIV equipped with a waterimmersion objective lens (UPlanSApo 60x/NA $=1.2$ ) and a dichroic mirror (DM405/473/559/635). The two fluorescence detection channels (Ch) were set to the following filters: Ch1: BP 400-440 (red pseudo color) for Lyso Tracker Blue DND-22 and Ch2: BP 610-710 (green pseudo color) for Cy5.

In the case of mitochondrial staining, 30 minutes before the acquisition of the fluorescence images, the medium was replaced with fresh medium containing Rhodamine123 (final concentration, $100 \mathrm{ng} / \mathrm{mL}$ ) and the cells were incubated in this solution. After this incubation, the cells were washed with the phenol red-free medium containing serum, and then observed by CLSM (LSM510 META; Carl Zeiss Co. Ltd., Jena, Germany). The cells were excited with a $488 \mathrm{~nm}$ light from an Ar laser for detecting Rhodamine 123 and a $633 \mathrm{~nm}$ light from a $\mathrm{He} / \mathrm{Ne}$ laser for detecting Cy5. Images were obtained using a LSM510 META equipped with an oil-immersion objective lens (Plan-Apochromat $63 \mathrm{x} / \mathrm{NA}=1.4$ ) and a dichroic mirror (HFT488/543/633). The two fluorescence detection channels (Ch) were set to the following filters: Ch1: BP 500-550 (red pseudo color) for rhodamine123 and Ch2: 665-719 (green pseudo color) for Cy5.

Fluorescence image processing for calculation of mitochondrial targeting activity 
Fluorescent and bright-field images for mitochondria that had been stained with Rhodamine123, were captured using an LSM510 META microscope, as shown in Figure 5A,B. Each 8-bit TIFF image was analyzed using the Image-Pro plus v.4.5.1.26 software (Media Cybernetics, Inc., Bethesda, MD) to quantify the total brightness of each region of interest. First, the yellow pixel areas where oligo DNA (green pseudo color) co-localized with mitochondria (red pseudo color) were marked in each image. In some cases, it was difficult to distinguish the localization of the carrier between inside and outside the mitochondria. Therefore, criteria were introduced so as to classify the carrier cluster as being co-localized inside the mitochondria when more than $50 \%$ of the pixels of the oligo DNA were mitochondria targeted positive (defined as green fluorescent values larger than 130). We also counted only clusters with sizes of more than 10 pixels of the signal area. The yellow and green pixel areas of each cluster in the mitochondria, $\mathrm{s}_{\mathrm{i}}$ (mt) and the outside of mitochondria, $s_{i}$ (out), were separately summed for each image, and are denoted as $S^{\prime}{ }_{Z=j}(m t), S^{\prime}{ }_{Z=j}($ out $)$, respectively. The values of $S^{\prime}{ }_{Z=j}(m t)$ and $S^{\prime}{ }_{Z=j}($ out $)$ in each image were further summed and are denoted as $\mathrm{S}(\mathrm{mt})$ and $\mathrm{S}($ out $)$, respectively. These parameters represent the total amount of oligo DNA inside and outside the mitochondria in the total cell. Furthermore, the total area of the oligo DNA, denoted as S(tot), was calculated by integrating the $\mathrm{S}(\mathrm{mt})$ and $\mathrm{S}$ (out). This value represents the total cellular uptake of oligo DNA. The mitochondrial targeting activity was calculated as $\mathrm{S}(\mathrm{mt})$ divided by $\mathrm{S}(\mathrm{tot})$.

\section{Evaluation of cell viability}

HeLa cells ( 4 × $10^{4}$ cells) were seeded on a 24 -well plate (Corning) with DMEM, containing $10 \%$ FBS, under an atmosphere of $5 \% \mathrm{CO}_{2} /$ air at $37^{\circ} \mathrm{C}$ for $24 \pm 3 \mathrm{hr}$. The cells were washed with PBS (-) before incubation with the carriers (final concentration of oligo DNA, $80 \mathrm{nM}$ ). The cells were then incubated in serum-free medium for $1 \mathrm{hr}$ under an atmosphere of $5 \% \mathrm{CO}_{2} /$ air at $37^{\circ} \mathrm{C}$. After replacing the medium with fresh DMEM containing $10 \%$ serum, the cells were incubated until used in assays. Cell viabilities were measured using a Cell Proliferation Assay System with a Tetra Color ONE (Seikagaku Biobusiness, Tokyo, Japan), according to the instructions provided by the manufacturer. Briefly, tetrazolium (WST-8) was added to a final volume of $275 \mu \mathrm{L} /$ well. After a 1-hour incubation, the absorbance at $450 \mathrm{~nm}$ was measured by means of an EnSpireTM 2300 Multilabel Reader (PerkinElmer, Inc.; Waltham, MA, USA). Cell viability was calculated as follows;

$$
\text { Cell viability }(\%)=\mathrm{A}_{\mathrm{T}} / \mathrm{A}_{\mathrm{U}} \times 100
$$

where $A_{T}$ and $A_{U}$ represent the absorbance at $450 \mathrm{~nm}$ for treated and untreated cells, respectively.

Observations of cells after staining mitochondria with Rhodamine123 and MitoTracker Deep Red 633.

HeLa cells ( 4 x $10^{4}$ cells) were cultured in $35 \mathrm{~mm}$ dishes with DMEM, which contained 10\% FBS, under an atmosphere of $5 \% \mathrm{CO}_{2} /$ air at $37^{\circ} \mathrm{C}$ for $24 \pm 3 \mathrm{hr}$. The DF-MITO-Porter or the 
conventional MITO-Porter encapsulating oligo DNA were added to the HeLa cells (final concentration of oligo DNA, $80 \mathrm{nM}$ ). The cells were then incubated in phenol red-free medium without serum under an atmosphere of $5 \% \mathrm{CO}_{2} /$ air at $37^{\circ} \mathrm{C}$. After a 1 -hr incubation, the medium was replaced with fresh phenol red-free medium containing serum, and the cells were incubated in the absence of the carriers for $2 \mathrm{hr}$. The cells were treated with Rhodamine123 and MitoTracker Deep Red 633 to stain mitochondria, and were then observed by CLSM. Rhodamine 123, a conventional fluorescent stain for mitochondria, is easily washed out of cells once the mitochondria experience a loss in membrane potential. On the other hand, MitoTracker Deep Red 633 is also selective for mitochondria and the stain is retained, even when mitochondrial membrane potential is lost. This experiment allowed us to evaluate the extent to which the carriers affected mitochondrial membrane potential.

As described above, mitochondria were stained with Rhodamine123. After washing the cells with phenol red-free medium containing serum, the medium was replaced with fresh medium containing MitoTracker Deep Red 633 (final concentration, $100 \mathrm{nM}$ ) and the cells were incubated in this solution for $30 \mathrm{~min}$. After this incubation, the cells were washed with phenol red-free medium containing serum, and then observed by CLSM (FV10i-LIV). The cells were excited with a $473 \mathrm{~nm}$ light for detecting Rhodamine123 and a $635 \mathrm{~nm}$ light for detecting MitoTracke Deep Red 633 from a LD laser. Images were obtained using a FV10i-LIV equipped with a waterimmersion objective lens (UPlanSApo 60x/NA $=1.2$ ) and a dichroic mirror (DM405/473/559/635). The two fluorescence detection channels (Ch) were set to the following filters: Ch1: BP 490-540 (green color) for Rhodamine123 and Ch2: BP 660-710 (red pseudo color) for MitoTracker Deep Red. When mitochondrial membrane potential was depolarized, the cells were treated with carbonyl cyanide 4-(trifluoromethoxy)phenylhydrazone (FCCP, Sigma), a mitochondrial uncoupler, before observation (final concentration of FCCP, $50 \mu \mathrm{M}$ ). 


\section{Results}

\section{Construction of the DF-MITO-Porter encapsulating oligo DNA}

In this study, we attempted to achieve mitochondrial-targeted DNA delivery by appropriately regulating intracellular trafficking. To accomplish this, we designed an intelligent nano device, by integrating high-density R8-modification to enhance cellular uptake, incorporating an endosomefusogenic lipid envelope for endosomal escape and the MITO-Porter for delivering a macromolecule to mitochondria (Fig. 1). The construction of the DF-MITO-Porter encapsulating oligo DNA requires the following three steps (Fig. S1): (1) the preparation of nanoparticles containing oligo DNA; (2) coating of the nanoparticles with a mitochondria-fusogenic envelope to produce a conventional MITO-Porter; (3) further coating with an endosome-fusogenic envelope in a step-wise manner. We first determined the optimal conditions for complexation between oligo DNA and STR-R8 (Futaki et al. 2001). We mixed oligo DNA with STR-R8 at several nitrogen / phosphate (N/P) ratios to form nanoparticles and then determined their diameters and $\zeta$-potentials (Fig. 2). Figure 2A shows data for the diameters of the complexes, which were approximately 50 $\mathrm{nm}$, when produced using N/P ratios higher than 2.5 . Figure $2 \mathrm{~B}$ shows the $\zeta$ potentials for the complexes, the charges of which changed from minus to plus with increasing N/P ratios. In this study, we used positively charged particles formed at an N/P molar ratio of 3.0 for the preparation of the DF-MITO-Porter.

For the packaging of oligo DNA nanoparticle, the inner envelope had a mitochondriafusogenic composition [DOPE/SM/CHEMS/STR-R8 (9:2:1:1, molar ratio)] (Yamada et al. 2008; Yamada et al. 2011), while the outer envelope had a endosome-fusogenic composition [DOPE/PA/STR-R8 (7:2:1, molar ratio)] (El-Sayed et al. 2008; Akita et al. 2009). The positively charged nanoparticles (about $15 \mathrm{mV}$ ) were coated with an anionic lipid film by the hydration method. The resulting vesicles (about $-40 \mathrm{mV}$ ) were then modified with R8 to reverse the surface charge, to produce a conventional MITO-Porter (about $50 \mathrm{mV}$ ). The conventional MITO-Porter was next packaged with outer envelopes through the membrane fusion of neighboring small unilamellar vesicles (SUVs), triggered by the assembly of negatively charged SUVs around the positively charged MITO-Porter, as shown in our previously report (Akita et al. 2009; Yamada et al. 2011). The resulting vesicles (about $-40 \mathrm{mV}$ ) were then modified with $\mathrm{R} 8$ to reverse the surface charge (about $60 \mathrm{mV}$ ). The conversion of the $\zeta$ potential in the coating process strongly suggests that the complexed nanoparticles of oligo DNA were encapsulated in a stepwise manner. The physiochemical properties of the carriers and the nanoparticle of oligo DNA are summarized in Table 1. We also characterized the intermediate vesicles (Table S1).

\section{Evaluation of the cellular uptake of the oligo DNA encapsulated carriers}


The first barrier to intracellular targeting is the plasma membrane. To overcome this barrier, we used R8 as a cellular uptake device. R8 (Futaki et al. 2001; Nakase et al. 2004) is a synthetic peptide that mimics the trans-activating transcriptional activator (TAT) derived from the human immunodeficiency virus (Joliot and Prochiantz 2004; Schwarze et al. 1999). To evaluate the cellular uptake efficiency of the carriers, Cy5-labelled oligo DNA encapsulated carriers were added to HeLa cells, and the fluorescent intensity of the oligo DNA uptaken by cells were measured by flow cytometry (Fig. 3). The cellular uptake of the carriers was calculated as the mean fluorescence intensity of the flow cytometry histogram shown in Figure 3A. The results showed that both the DF-MITO-Porter and the conventional MITO-Porter significantly enhanced the cellular uptake of the oligo DNA compared to that of an oligo DNA nanoparticle without lipid envelope (Fig. 3B). We also observed the same tendency for cellular uptake, when the carriers labeled with NBD-DOPE lipids were used to evaluate the cellular uptake of carriers themselves (Fig. S2).

It has been reported that macropinocytosis is the major entrance pathway for high-density R8-modified liposomes rather than clathrin-mediated endocytosis, as is operative in the case of cationic liposomes, and that the lipid composition has no effect on the internalization mechanism (Khalil et al. 2006). Thus, we assumed that the efficiencies of cellular uptake of DF-MITO-Porter and conventional MITO-Porter would be similar, although the lipid composition in the outer envelopes was different. The $\zeta$ potentials of the DF-MITO-Porter and conventional MITO-Porter were positive (Table 1), suggesting that the surface of the carrier had been modified with R8, as shown in Figure S1. The positively charged carriers would be expected to have easy access to the cellular membrane, which is negatively charged via electrostatic interactions. Based on these results, we concluded that the nanoparticle packaging of oligo DNA in a high-density R8-modified lipid envelope would be useful for the cellular uptake of oligo DNA.

\section{Intracellular observation of carriers after staining endosome}

After the carriers become internalized by the cell, they become entrapped in endocytic vesicles, unless they are equipped with a device that permits them to readily escape. The carriers must escape from the endosomes to the cytosol, in order to reach their final destination (mitochondria). We performed the intracellular observation of the carriers using CLSM to verify that the intracellular trafficking of the carrier could be regulated for efficient mitochondrial delivery. To check endosomal escape efficiency, we observed the intracellular trafficking of the carriers after staining the endosomes (Fig. 4). In the case of the DF-MITO-Porter, numerous green dots were observed in cells, and most of these were outside the acidic compartment (Fig. 4A), suggesting that the carriers had been released from the endosomes to the cytosol. When the conventional MITO-Porter was used, green dots were observed in the cells, but some of the oligo DNA was localized in acidic compartments, as evidenced by the location of the yellow dots (Fig. 4B). These results show that the DF-MITO-Porter had a higher endosomal escape efficiency than that of the 
conventional MITO-Porter, although the cellular uptake efficiency of the carriers were similar, as shown in Figure 3 and Figure S2.

In this experiment, we used the lipid composition [DOPE/PA/STR-R8 (7:2:1, molar ratio)] for the outer envelope, because we demonstrated that this lipid composition permitted the particles to fuse with the endosome membrane in living cells (El-Sayed et al. 2008; Akita et al. 2009). In the previous study, the lipid composition adequate for fusion to the endosome-membrane was screened by monitoring the cancellation of FRET between donor and acceptor fluorophores, modified on the surface of liposomes with cultured cells at $\mathrm{pH}$ 6.5, which mimics the acidic environment of the endosome. The dual labeled liposomes were also useful probes for visualizing the intracellular fusion event, in which FRET activity can be monitored by spectral imaging. The analysis demonstrated that liposomes prepared with the lipid composition [DOPE/PA/STR-R8 (7:2:1, molar ratio)] drastically enhanced endosomal escape. In the present study, we were also able to show that the optimal endosome-fusogenic composition would be useful for mitochondrial delivery as the outer envelope of the DF-MITO-Porter.

\section{Intracellular observation after staining mitochondria and the evaluation of mitochondrial targeting activity}

The final process for mitochondrial delivery includes targeting the carriers to mitochondria and the introduction of the cargos into the mitochondrial compartment. For the former, R8 was chosen as a mitochondrial targeting device, because recent studies reported that cell permeable peptides such as TAT and R8 have the ability to deliver a certain cargo to mitochondria (Asoh et al. 2002; Del Gaizo and Payne 2003; Yamada and Harashima 2008). The introduction of cargos into mitochondria by the MITO-Porter system was found to greatly assist delivery through the mitochondrial membrane via membrane fusion (Yamada et al. 2008). We observed the intracellular trafficking of the carriers after staining mitochondria (Fig. 5A,B). Several yellow clusters were observed, indicating that the Cy5 in the oligo DNAs (green pseudo color) were colocalized with mitochondria (red pseudo color) and observed as a yellow signal. It was confirmed that the DF-MITO-Porter accumulated in mitochondria more efficiently compared to the conventional MITO-Porter (Fig. 5A,B). We next evaluated the mitochondrial targeting activity based on images observed by CLSM (see Materials and methods for details). For this evaluation, we applied a confocal image-assisted integrated quantification method (Akita et al. 2004; Hama et al. 2006) to calculate the mitochondrial targeting activity. This value represents ratio of oligo DNA localized in mitochondria within a single cell. Consequently, the mitochondrial targeting activity of the DF-MITO-Porter was significantly higher than the corresponding value for the conventional MITO-Porter (Fig. 5C).

In a previous study, we constructed a DF-MITO-Porter encapsulating DNase I protein, and evaluated the pharmacological effect of the carrier on mitochondria (Yamada et al. 2011). As a 
result, the mitochondrial delivery of DNase I protein by the DF-MITO-Porter drastically decreased mtDNA-levels. We also performed intracellular observations to evaluate the fraction of mitochondrial targeted positive cells, where carriers were colocalized with mitochondria in at least one Z-series image. Consequently, the value for the DF-MITO-Porter (83\%) was considerably higher than the corresponding value for the conventional MITO-Porter (25\%) (Yamada et al. 2011). We presumed that the endosomal fusogenic outer envelope of the DF-MITO-Porter mainly contributed to the increase in the fraction of mitochondrial targeted positive cells, where the carrier was delivered to mitochondria escaping from lysosome-degradation, and this would influence the pharmacological effect on mitochondria of the total cell population.

The fraction of mitochondrial targeted positive cells provided information regarding the mitochondrial delivery of carriers to the total population of cells. However, it was not possible to evaluate the mitochondrial selectivity of carriers in a single cell. Mitochondrial delivery requires the regulation of complicated intracellular processes. In this study, we attempted to evaluate these intracellular multiple processes i.e., (i) cellular uptake, (ii) endosomal escape and (iii) mitochondrial delivery, in order to clarify the rate limiting step of the mitochondrial-targeted DNA delivery using the carrier. Flow cytometry analysis indicated that the nanoparticle packaging of oligo DNA with a high-density R8-modified lipid envelope would be useful for the cellular uptake of oligo DNA. Intracellular observation using CLSM confirmed that a multi-layered structure with endosomal fusogenic activity assisted the endosomal escape process. As a result, the DF-MITOPorter was delivered to mitochondria more effectively than a conventional MITO-Porter. These results support the conclusions reached in a previous study that by improving endosomal escape the fraction of mitochondrial targeted positive cells is increased, resulting in a sufficient pharmacological effect on mitochondria, when DNase I protein was used as the cargo of the DFMITO-Porter (Yamada et al. 2011).

On the other hand, a quantification analysis of mitochondrial targeting activity disclosed the fact that approximately $80 \%$ of the oligo DNA remained outside mitochondria in the case of mitochondrial delivery by the DF-MITO-Porter (Fig. 5C). Based on these results, we conclude that the rate-limiting step in mitochondrial delivery using the carriers appears to be the mitochondrial targeting process from the cytosol. These analyses indicated that the use of the DF-MITO-Porter results in an increase in the fraction of mitochondrial targeted positive cells, and that the intracellular trafficking of carriers could be insufficiently controlled. A previous investigation showed that the mitochondrial binding activity of liposomes increased significantly after R8modification (Yamada and Harashima 2008). This result indicates that the addition of a cationic peptide, R8, to the liposome surface enhances mitochondrial binding, probably because a high negative potential is maintained. Thus, we used R8 as a mitochondrial targeting device, and we equipped inner envelope in the DF-MITO-Porter with R8. Unfortunately, R8-modified carries would be unable to sufficiently control the mitochondrial targeting process from the cytosol in living cells, different from when isolated mitochondria are being used.

It was recently reported that the conjugation of MTS achieved selective mitochondrial delivery, not via electrostatic interaction but via the mitochondrial import machinery (Flierl et al. 
2003; Schatz 1996), but this strategy failed in cases of macromolecules and hydrophobic molecules (Endo et al. 1995; Esaki et al. 1999; Gruhler et al. 1995). Moreover, this strategy should include the ability for cytoplasmic delivery, because MTS itself cannot be internalized through the cellular membrane. In the future, we plan to integrate an MTS and a DF-MITO-Porter system to achieve the effective delivery of DNA to mitochondria in living cells.

\section{Evaluation of cell viability and mitochondrial membrane potential after incubation with the oligo DNA encapsulated carriers}

The possibility that the DF-MITO-Porter might induce cell toxicity cannot be excluded, since this system fuses with mitochondria, which play a role in the homeostasis of vital physiological functions. Therefore, we investigated cell viabilities at different times after incubation with the carriers. In this experiment, we prepared the DF-MITO-Porter and a conventional MITO-Porter encapsulating oligo DNA, and then evaluated cell viabilities at several points after incubation with the carriers (Fig. 6). No cell toxicity was observed at any time for both the DF-MITO-Porter (open circles in Fig. 6) and the conventional MITO-Porter (open triangles in Fig. 6). The conditions used were similar to those of the other experiments in this study, where oligo DNA was added to cells (final concentration, $80 \mathrm{nM}$ ). Based on these results, we conclude that the above carriers were not toxic to cells, and could be safely used in a mitochondrial delivery system.

We also evaluated the mitochondrial membrane potentials after incubation with the carriers. In this experiment, we stained mitochondria with Rhodamine123 and MitoTracker Deep Red 633, and then observed the cells by CLSM (Fig. 7, Fig. S3 in Supplemental Material). Rhodamine 123 stains only intact mitochondria with a high membrane potential. On the other hand, MitoTracker Deep Red 633 can stain mitochondria, even when membrane potential decreases. In the case of both the DF-MITO-Porter (G-I in Fig. 7, A-C in Fig. S3) and the conventional MITO-Porter (J-L in Fig. 7, D-F in Fig. S3), mitochondria were extensively stained with Rhodamine123 at a level comparable to non-treatment cells (A-C in Fig. 7, G-I in Fig. S3). Moreover, it was confirmed that mitochondrial images stained by Rhodamine123 and MitoTracker Deep Red 633 had nearly merged, and were observed as yellow signal. This suggests that most of the mitochondria possessed a high membrane potential, when the cells were incubated with carriers in this condition (final concentration of oligo DNA, $80 \mathrm{nM}$ ). We also confirmed that Rhodamine123 stained mitochondria incompletely, when the mitochondrial membrane potential was depolarized, as evidenced when the cells were treated with FCCP (a mitochondrial uncoupler) (D-F in Fig. 7). In such severe conditions, Rhodamine123 failed to sufficiently stain mitochondria.

These results indicate that the use of the carriers do not decrease the mitochondrial membrane potential significantly, compared to FCCP treatment. However, this result does not determine whether mitochondria that fused with the carriers have enough membrane potential. The possibility that the DF-MITO-Porter with high mitochondrial targeting activity would decrease the mitochondrial membrane potential cannot be excluded, since this system fuses with the mitochondria. Thus, the intracellular observation after staining mitochondria with Rhodamine123 (Fig. 5) provided the information concerning targeting activity of carriers to mitochondria with 
enough membrane potentials. In the future, we attempt to investigate that the mitochondria efficiently targeted with the carriers maintain functionality.

\section{Conclusion}

In this study, we constructed an oligo DNA encapsulated DF-MITO-Porter in which high-density R8-modification is incorporated for cellular uptake, an endosome-fusogenic lipid envelope for endosomal escape and a MITO-Porter for delivering a macromolecule to mitochondria. We then compared the intracellular multiple processes between the DF-MITO-Porter and a conventional MITO-Porter. Flow cytometry analysis indicated that a high-density R8-modified lipid envelope would be useful for achieving cellular uptake. Intracellular observation using CLSM showed that the DF-MITO-Porter could escape from endosome more effectively than a conventional MITOPorter, resulting in higher mitochondrial targeting activity. On the other hand, a quantification analysis of mitochondrial targeting activity suggests that the rate-limiting step for mitochondrial delivery using the carrier is the mitochondrial targeting process from the cytosol. Furthermore, the evaluation of cell cytotoxicity showed that the DF-MITO-Porter was not toxic to the cells. Future studies will involve attempts to improve the DF-MITO-Porter in terms of mitochondrial targeting activity. Studies related to this issue are currently in progress.

\section{Acknowledgements}

This work was supported, in part by, the Program for Promotion of Fundamental Studies in Health Sciences of the National Institute of Biomedical Innovation, Japan (NIBIO), a Grant-in-Aid for Young Scientists (A) and a Grant-in-Aid for Scientific Research (S) from the Ministry of Education, Culture, Sports, Science and Technology of Japanese Government (MEXT). We also thank Dr. Milton Feather for his helpful advice in writing the manuscript. 


\section{References}

Akita H, Ito R, Khalil IA, Futaki S, Harashima H (2004) Quantitative three-dimensional analysis of the intracellular trafficking of plasmid DNA transfected by a nonviral gene delivery system using confocal laser scanning microscopy. Mol Ther 9 (3):443-451

Akita H, Kudo A, Minoura A, Yamaguti M, Khalil IA, Moriguchi R, Masuda T, Danev R, Nagayama K, Kogure K, Harashima H (2009) Multi-layered nanoparticles for penetrating the endosome and nuclear membrane via a step-wise membrane fusion process. Biomaterials 30 (15):2940-2949

Asoh S, Ohsawa I, Mori T, Katsura K, Hiraide T, Katayama Y, Kimura M, Ozaki D, Yamagata K, Ohta S (2002) Protection against ischemic brain injury by protein therapeutics. Proc Natl Acad Sci U S A 99 (26):17107-17112

Chen Z, Felsheim R, Wong P, Augustin LB, Metz R, Kren BT, Steer CJ (2001) Mitochondria isolated from liver contain the essential factors required for RNA/DNA oligonucleotidetargeted gene repair. Biochem Biophys Res Commun 285 (2):188-194

D'Souza GG, Boddapati SV, Weissig V (2005) Mitochondrial leader sequence--plasmid DNA conjugates delivered into mammalian cells by DQAsomes co-localize with mitochondria. Mitochondrion 5 (5):352-358

D'Souza GG, Rammohan R, Cheng SM, Torchilin VP, Weissig V (2003) DQAsome-mediated delivery of plasmid DNA toward mitochondria in living cells. J Control Release 92 (12):189-197

Del Gaizo V, Payne RM (2003) A novel TAT-mitochondrial signal sequence fusion protein is processed, stays in mitochondria, and crosses the placenta. Mol Ther 7 (6):720-730

El-Sayed A, Khalil IA, Kogure K, Futaki S, Harashima H (2008) Octaarginine- and octalysinemodified nanoparticles have different modes of endosomal escape. J Biol Chem 283 (34):23450-23461

Endo T, Nakayama Y, Nakai M (1995) Avidin fusion protein as a tool to generate a stable translocation intermediate spanning the mitochondrial membranes. J Biochem (Tokyo) 118 (4):753-759

Esaki M, Kanamori T, Nishikawa S, Endo T (1999) Two distinct mechanisms drive protein translocation across the mitochondrial outer membrane in the late step of the cytochrome b(2) import pathway. Proc Natl Acad Sci U S A 96 (21):11770-11775

Flierl A, Jackson C, Cottrell B, Murdock D, Seibel P, Wallace DC (2003) Targeted delivery of DNA to the mitochondrial compartment via import sequence-conjugated peptide nucleic acid. Mol Ther 7 (4):550-557

Futaki S, Ohashi W, Suzuki T, Niwa M, Tanaka S, Ueda K, Harashima H, Sugiura Y (2001) Stearylated arginine-rich peptides: a new class of transfection systems. Bioconjug Chem 12 (6): 1005-1011

Goto Y, Nonaka I, Horai S (1990) A mutation in the tRNA(Leu)(UUR) gene associated with the MELAS subgroup of mitochondrial encephalomyopathies. Nature 348 (6302):651-653

Gruhler A, Ono H, Guiard B, Neupert W, Stuart RA (1995) A novel intermediate on the import pathway of cytochrome b2 into mitochondria: evidence for conservative sorting. Embo $\mathrm{J}$ 14 (7):1349-1359

Hama S, Akita H, Ito R, Mizuguchi H, Hayakawa T, Harashima H (2006) Quantitative comparison of intracellular trafficking and nuclear transcription between adenoviral and lipoplex systems. Mol Ther 13 (4):786-794

Holt IJ, Harding AE, Morgan-Hughes JA (1988) Deletions of muscle mitochondrial DNA in patients with mitochondrial myopathies. Nature 331 (6158):717-719

Holt IJ, Harding AE, Petty RK, Morgan-Hughes JA (1990) A new mitochondrial disease associated with mitochondrial DNA heteroplasmy. Am J Hum Genet 46 (3):428-433

James AM, Sharpley MS, Manas AR, Frerman FE, Hirst J, Smith RA, Murphy MP (2007) Interaction of the mitochondria-targeted antioxidant MitoQ with phospholipid bilayers and ubiquinone oxidoreductases. J Biol Chem 282 (20):14708-14718

Joliot A, Prochiantz A (2004) Transduction peptides: from technology to physiology. Nat Cell Biol 6 (3):189-196

Khalil IA, Kogure K, Futaki S, Harashima H (2006) High density of octaarginine stimulates macropinocytosis leading to efficient intracellular trafficking for gene expression. J Biol Chem 281 (6):3544-3551

Mukhopadhyay A, Weiner H (2007) Delivery of drugs and macromolecules to mitochondria. Adv Drug Deliv Rev 59 (8):729-738. doi:S0169-409X(07)00094-4 [pii] 
10.1016/j.addr.2007.06.004

Nakamura Y, Kogure K, Yamada Y, Futaki S, Harashima H (2006) Significant and prolonged antisense effect of a multifunctional envelope-type nano device encapsulating antisense oligodeoxynucleotide. J Pharm Pharmacol 58 (4):431-437. doi:10.1211/jpp.58.4.0002

Nakase I, Niwa M, Takeuchi T, Sonomura K, Kawabata N, Koike Y, Takehashi M, Tanaka S, Ueda K, Simpson JC, Jones AT, Sugiura Y, Futaki S (2004) Cellular uptake of argininerich peptides: roles for macropinocytosis and actin rearrangement. Mol Ther 10 (6):10111022

Schatz G (1996) The protein import system of mitochondria. J Biol Chem 271 (50):31763-31766

Schwarze SR, Ho A, Vocero-Akbani A, Dowdy SF (1999) In vivo protein transduction: delivery of a biologically active protein into the mouse. Science 285 (5433):1569-1572

Seibel P, Trappe J, Villani G, Klopstock T, Papa S, Reichmann H (1995) Transfection of mitochondria: strategy towards a gene therapy of mitochondrial DNA diseases. Nucleic Acids Res 23 (1):10-17

Shoffner JM, Lott MT, Lezza AM, Seibel P, Ballinger SW, Wallace DC (1990) Myoclonic epilepsy and ragged-red fiber disease (MERRF) is associated with a mitochondrial DNA tRNA(Lys) mutation. Cell 61 (6):931-937

Taylor RW, Chinnery PF, Turnbull DM, Lightowlers RN (1997) Selective inhibition of mutant human mitochondrial DNA replication in vitro by peptide nucleic acids. Nat Genet 15 (2):212-215

Tuppen HA, Blakely EL, Turnbull DM, Taylor RW (2010) Mitochondrial DNA mutations and human disease. Biochim Biophys Acta 1797 (2):113-128. doi:S0005-2728(09)00261-8 [pii]

10.1016/j.bbabio.2009.09.005

Vestweber D, Schatz G (1989) DNA-protein conjugates can enter mitochondria via the protein import pathway. Nature 338 (6211):170-172

Wallace DC (2005) The mitochondrial genome in human adaptive radiation and disease: on the road to therapeutics and performance enhancement. Gene 354:169-180

Weissig V, D'Souza GG, Torchilin VP (2001) DQAsome/DNA complexes release DNA upon contact with isolated mouse liver mitochondria. J Control Release 75 (3):401-408

Wipf P, Xiao J, Jiang J, Belikova NA, Tyurin VA, Fink MP, Kagan VE (2005) Mitochondrial targeting of selective electron scavengers: synthesis and biological analysis of hemigramicidin-TEMPO conjugates. J Am Chem Soc 127 (36):12460-12461

Yamada Y, Akita H, Kamiya H, Kogure K, Yamamoto T, Shinohara Y, Yamashita K, Kobayashi H, Kikuchi H, Harashima H (2008) MITO-Porter: A liposome-based carrier system for delivery of macromolecules into mitochondria via membrane fusion. Biochim Biophys Acta 1778 (2):423-432

Yamada Y, Furukawa R, Yasuzaki Y, Harashima H (2011) Dual Function MITO-Porter, a Nano Carrier Integrating Both Efficient Cytoplasmic Delivery and Mitochondrial Macromolecule Delivery. Mol Ther 19 (8):1449-1456. doi:10.1038/mt.2011.99

mt201199 [pii]

Yamada Y, Harashima H (2008) Mitochondrial drug delivery systems for macromolecule and their therapeutic application to mitochondrial diseases. Adv Drug Deliv Rev 60 (13-14):14391462

Yamada Y, Kogure K, Nakamura Y, Inoue K, Akita H, Nagatsugi F, Sasaki S, Suhara T, Harashima H (2005) Development of efficient packaging method of oligodeoxynucleotides by a condensed nano particle in lipid envelope structure. Biol Pharm Bull 28 (10):1939-1942

Zhang E, Zhang C, Su Y, Cheng T, Shi C (2011) Newly developed strategies for multifunctional mitochondria-targeted agents in cancer therapy. Drug Discov Today 16 (3-4):140-146. doi:S1359-6446(10)00844-5 [pii]

10.1016/j.drudis.2010.12.006 


\section{Figure Captions}

Fig. 1 Schematic diagram illustrating the intracellular trafficking events associated with the Dual Function (DF)-MITO-Porter and conventional MITO-Porter. Modification of the outer envelopesurface with a high density of octaarginine greatly assists in the efficient uptake of both the DFMITO-Porter and the conventional MITO-Porter by cells (1st step). The DF-MITO-Porter can efficiently escape from the endosome via membrane fusion, a process that is mediated by the outer endosome-fusogenic lipid membranes (2nd step), whereas the efficiency of escape for the conventional MITO-Porter is low. A greater fraction of the conventional MITO-Porter undergoes endosomal degradation compared with DF-MITO-Porter. Finally, the carrier fuses with the mitochondrial membrane to deliver cargos into mitochondria (3rd step). Thus, the DF-MITOPorter would be more efficient than the conventional MITO-Porter in the process of endosomal escape.

Fig. 2 Characteristics of nanoparticles of oligo DNA complexed with STR-R8. Oligo DNA was complexed with STR-R8 at various nitrogen phosphate (N/P) ratios, and their diameters (A) and $\zeta$ potentials (B) were then measured. Data are represented by the mean \pm S.E. $(n=3)$.

Fig. 3 Evaluation of the cellular uptake of carriers by flow cytometry. The histogram plot (A) shows the fluorescence intensities of the oligo DNA labeled with Cy5 taken up by cells after the transduction of the DF-MITO-Porter [green line], the conventional MITO-Porter [pink line] and nanoparticles of oligo DNA [blue line]. The cellular uptake of the oligo DNA (B). Data are represented as the mean \pm S.E. $(\mathrm{n}=3) . *$ Significant difference $(\mathrm{p}<0.05$ by one-way ANOVA, followed by Student-Newman-Keuls (SNK) test). MFI, mean fluorescence intensity.

Fig. 4 Intracellular observation after staining endosomes by CLSM. To evaluate the endosomal escape of the carrier, the intracellular trafficking of the carriers encapsulating oligo DNA labeled with Cy5 (green pseudo color) was visualized after staining endosomes with LysoTracker Blue DND-22 (red pseudo color). Note that oligo DNA appears as yellow clusters when it is localized in endosomes. A, DF-MITO-Porter; B, Conventional MITO-Porter. Scale bars, $10 \mu \mathrm{m}$. 
Fig. 5 Intracellular observation after staining mitochondria by CLSM and evaluation of mitochondrial targeting activity. To evaluate mitochondrial targeting activity, the intracellular trafficking of the carriers encapsulating oligo DNA labeled with Cy5 (green pseudo color) was observed after staining mitochondria with Rhodamine 123 (red pseudo color). Oligo DNA (green) was seen to co-localize with mitochondria (red), observed as a yellow signal in the merged images. A, DF-MITO-Porter; B, Conventional MITO-Porter. Scale bars, $10 \mu \mathrm{m}$. Based on images, the mitochondrial targeting activity was calculated (C). Data are represented as the mean \pm S.E. $(n=$ 10). Statistical analysis was by a two-tailed unpaired Student's t test $(\mathrm{p}=0.0032)$.

Fig. 6 Evaluation of cell viability. Cell viabilities were measured at $1 \mathrm{hr}, 3 \mathrm{hr}, 6 \mathrm{hr}$ and $24 \mathrm{hr}$ after incubation with DF-MITO-Porter (open circles), conventional MITO-Porter (open triangles) and naked oligo DNA (open squares). Data are represented by the mean \pm S.E. $(n=3-4)$.

Fig. 7 Observations of cells after staining mitochondria with Rhodamine123 and MitoTracker Deep Red 633. Carriers were added to HeLa cells, and mitochondria were then stained with Rhodamine123 (green color; A, D, G, J) and MitoTracker Deep Red 633 (red pseudo color; B, E, $\mathrm{H}, \mathrm{K})$. Merge images (C, F, I, L) indicate yellow signals when mitochondria stained with Rhodamine123 and MitoTracker Deep Red 633 are overlapped. Staining of mitochondria with Rhodamine 123 is dependent on the membrane potential, while MitoTracker Deep Red 633 can stain mitochondria even when membrane potential is lost. The Cells were observed using CLSM; A-C, Non-treatment; D-F, FCCP; G-I, DF-MITO-Porter; J-L, Conventional MITO-Porter. Scale bars, $20 \mu \mathrm{m}$. Note that FCCP is an uncoupler that is capable of depolarizing mitochondrial membranes.

Table 1 Characteristics of the carriers and nanoparticle of oligo DNA.

\begin{tabular}{lccc}
\hline & Diameter $(\mathbf{n m})$ & PDI & $\zeta$ potential $(\mathbf{m V})$ \\
\hline Nanoparticle of ODN & $51 \pm 2$ & $0.269 \pm 0.038$ & $20 \pm 2$ \\
Conventional MITO-Porter & $186 \pm 6$ & $0.246 \pm 0.028$ & $59 \pm 4$ \\
DF-MITO-Porter & $190 \pm 10$ & $0.276 \pm 0.034$ & $61 \pm 5$ \\
\hline
\end{tabular}

Diameter, polydispersity index (PDI) and $\zeta$ potential of carriers were measured. Data are represented by the mean \pm S.D. $(n=3-5)$. 
Fig. 1

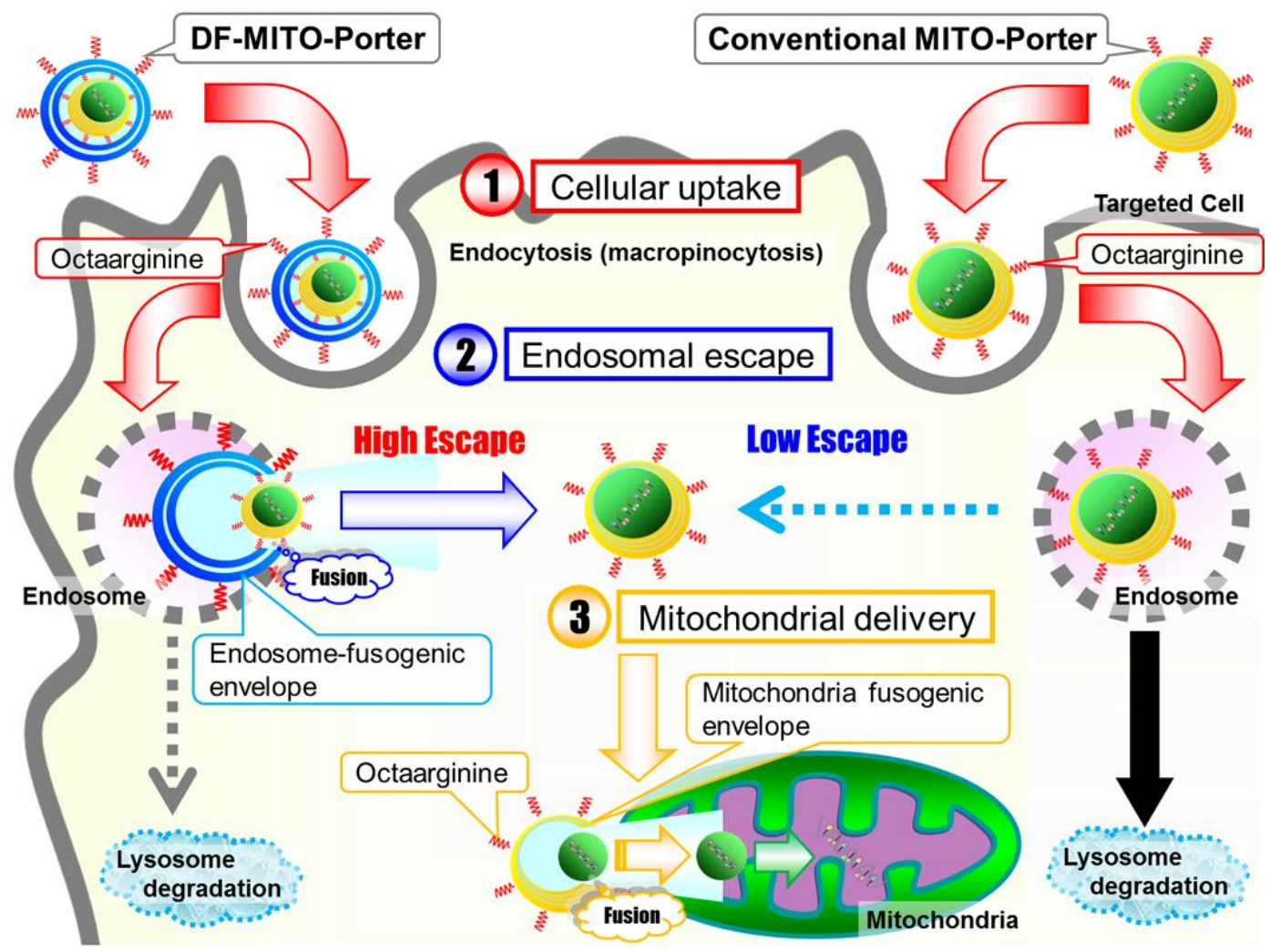


Fig. 2

A

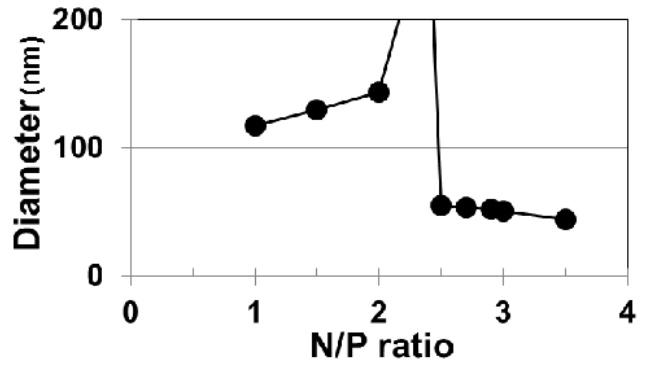

B

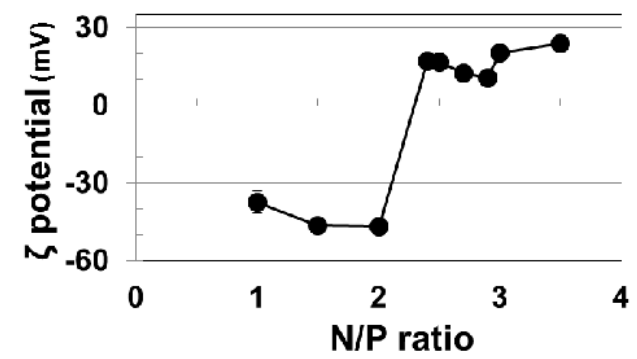


Fig. 3

A

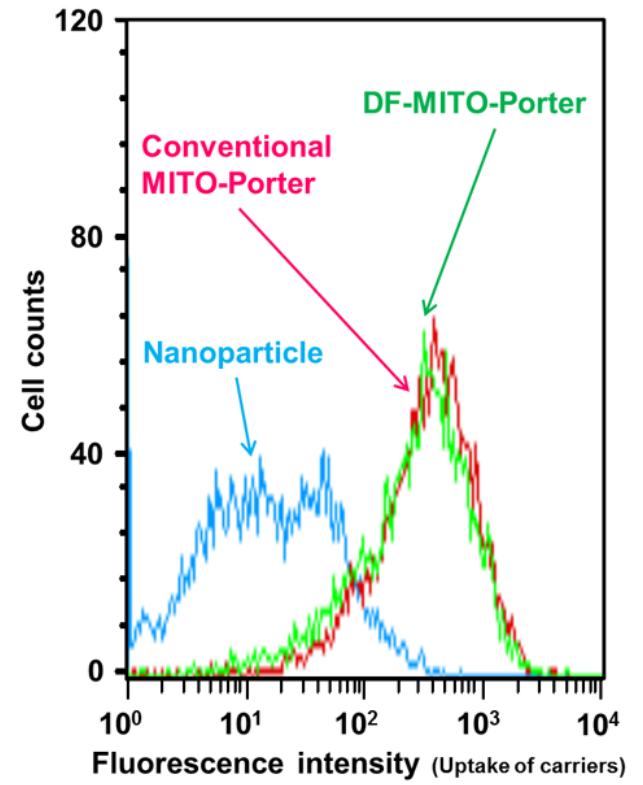

B

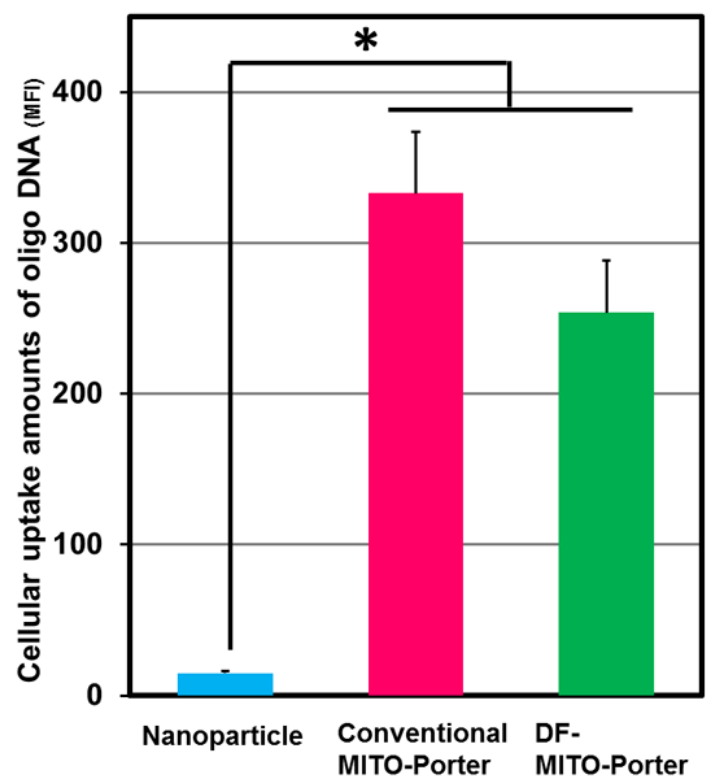


Fig. 4

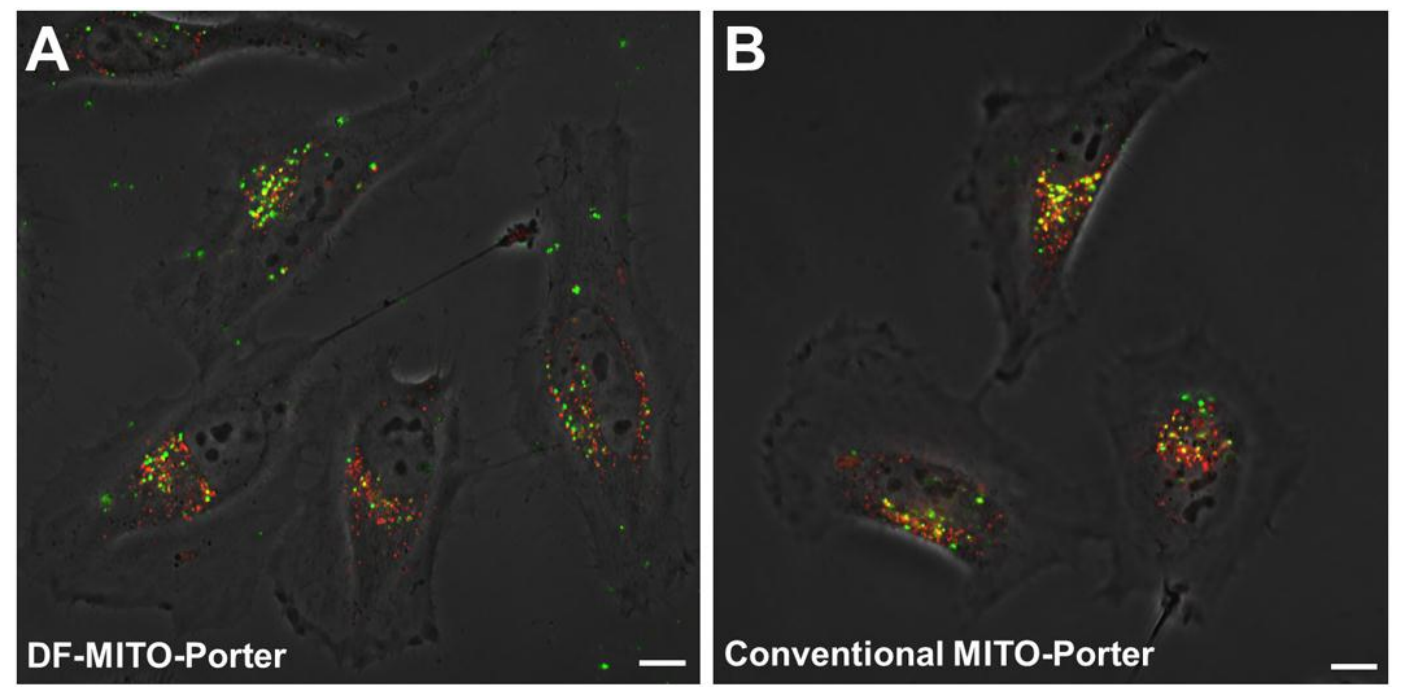


Fig. 5
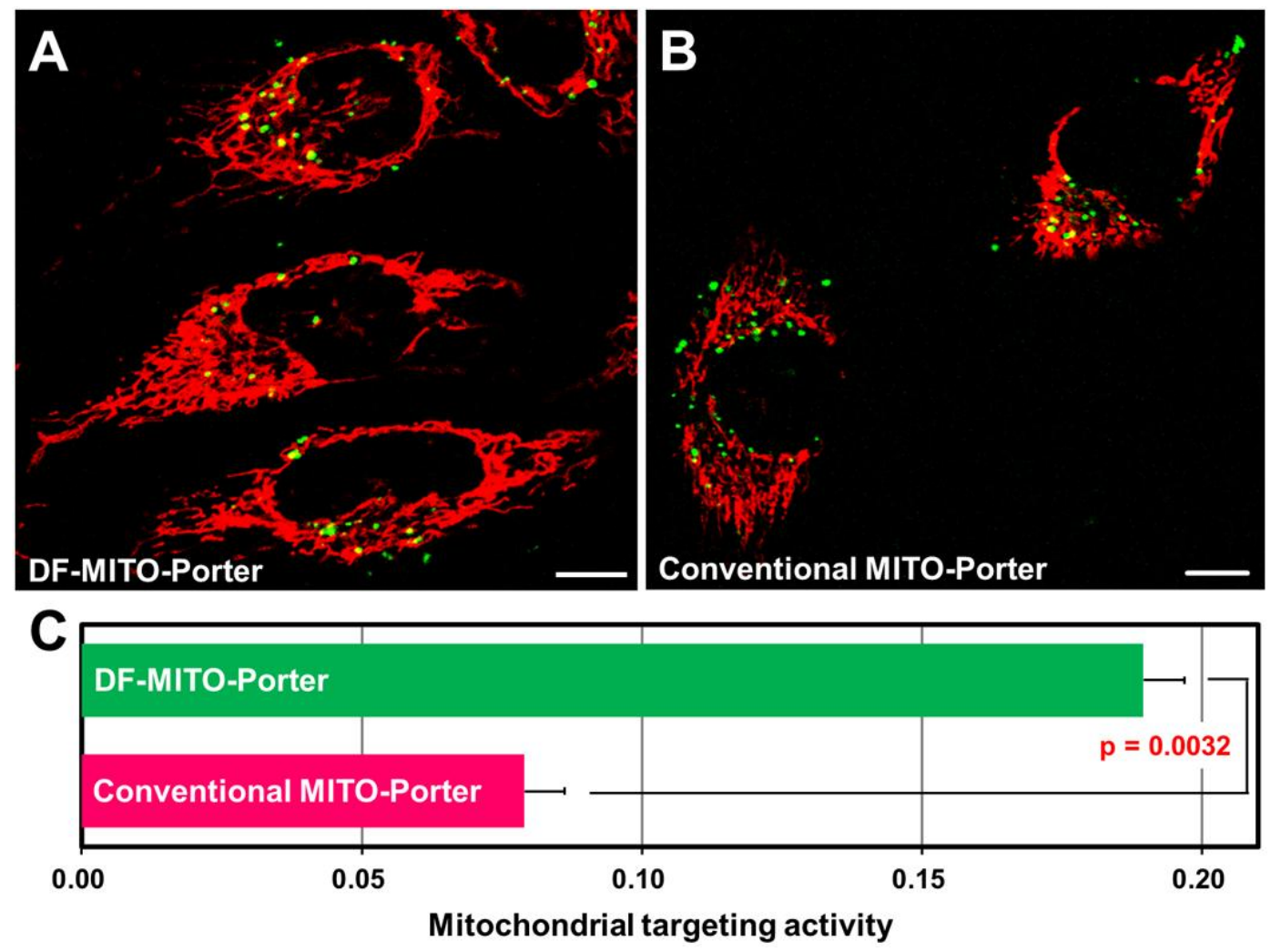
Fig. 6

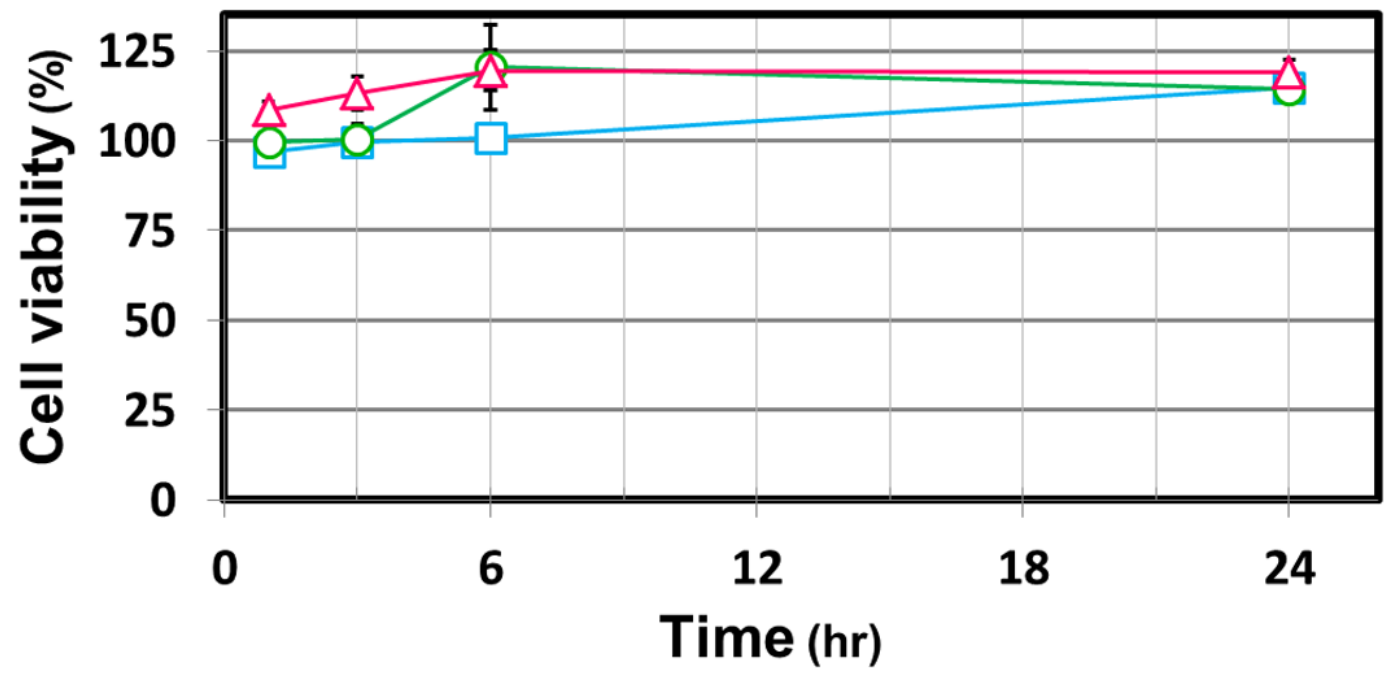


Fig. 7
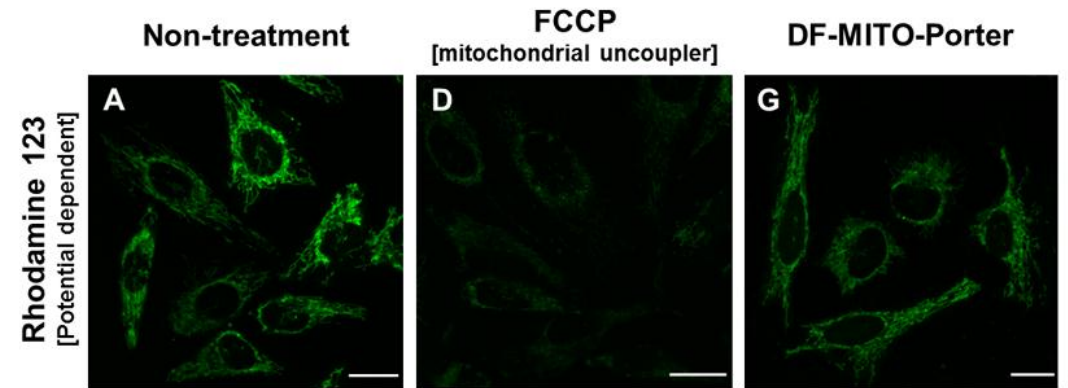

Conventional
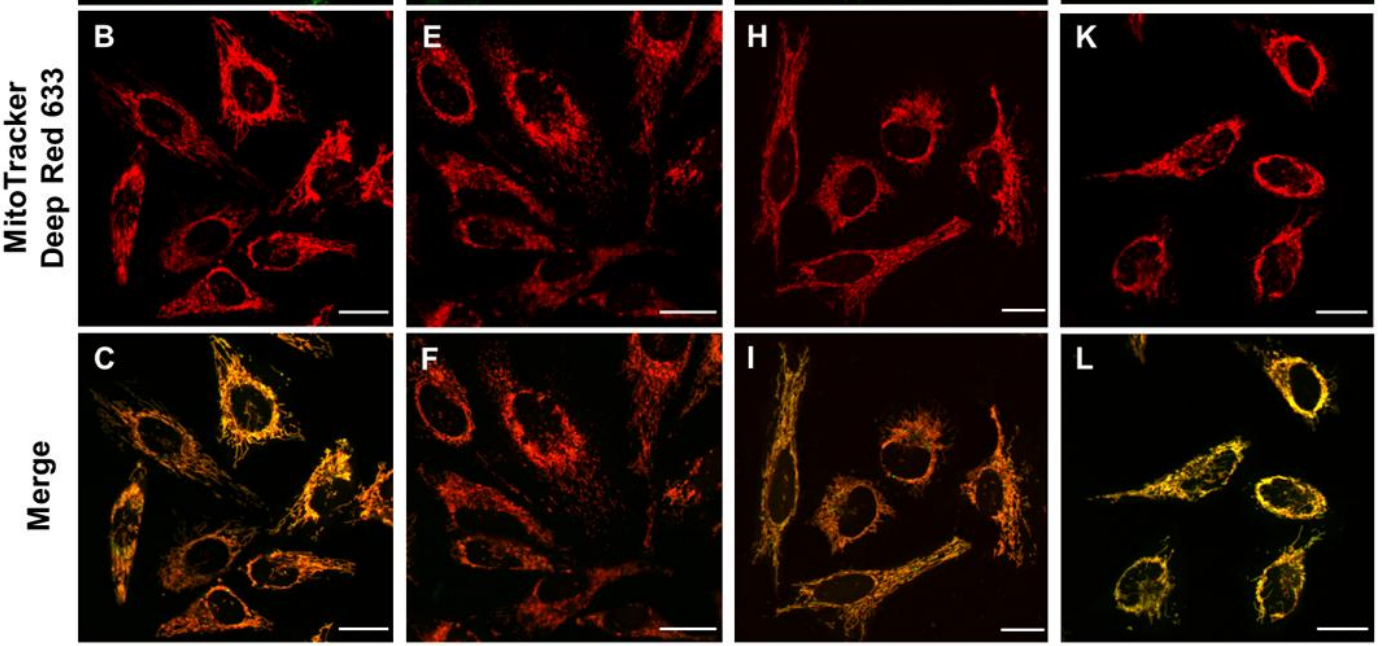


\section{Supplemental Material}

1. Physicochemical properties of intermediated vesicles of the DF-MITO-Porter encapsulating oligo DNA.

The construction of a DF-MITO-Porter encapsulating oligo DNA requires the three following steps: (1) preparation of nano particles containing oligo DNA; (2) coating of the nano particles with mitochondria-fusogenic envelope to produce a conventional MITO-Porter; (3) further coating with endosome-fusogenic envelope in a step-wise manner (Figure S1). The physiochemical properties of the intermediate vesicles of DF-MITO-Porter are summarized in Table S1.

Figure S1. Schematic diagram of the preparation of the DF-MITO-Porter and the conventional MITO-Porter

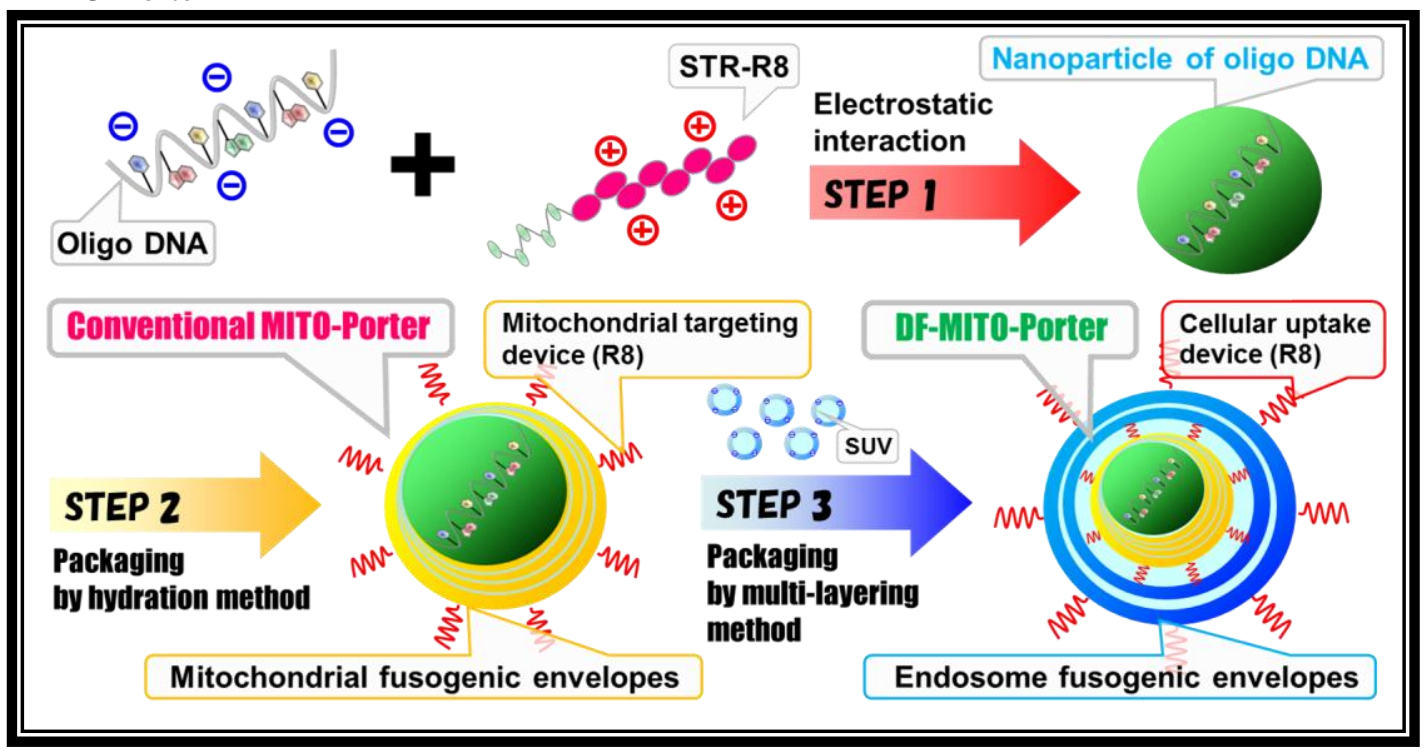

DF-MITO-Porter and conventional MITO-Porter encapsulating oligo DNA were constructed as shown in Figure S1. The major difference between the DF-MITO-Porter and the conventional MITO-Porter is that the DF-MITO-Porter contains an endosome fusogenic outer envelope. STR-R8, stearyl octaarginine; SUV, small unilamellar vesicle. 
Table S1. Characteristics of intermediated vesicles of the DF-MITO-Porter.

\begin{tabular}{|lcc|}
\hline & Diameter $(\mathrm{nm})$ & 弓 potential $(\mathrm{mV})$ \\
\hline MITO-Porter before modified with R8 & 187 & -38 \\
DF-MITO-Porter before modified with R8 & 173 & -39 \\
\hline
\end{tabular}

Diameter and $\zeta$ potential of carriers were measured. Data are represented by the mean $(n=1$ $3)$. 
2. Evaluation of the cellular uptake of carriers labeled with fluorescent probe by flow cytometry.

We utilized flow cytometry to evaluate the cellular uptake of the DF-MITO-Porter and the conventional MITO-Porter with inner lipid envelopes labeled with 1\% NBD-lipids (Fig. S2). The results showed that the DF-MITO-Porter was taken up by cells with approximately the same efficiency as the conventional MITO-Porter.

Figure S2. Evaluation of the cellular uptake of carriers by NBD lipids

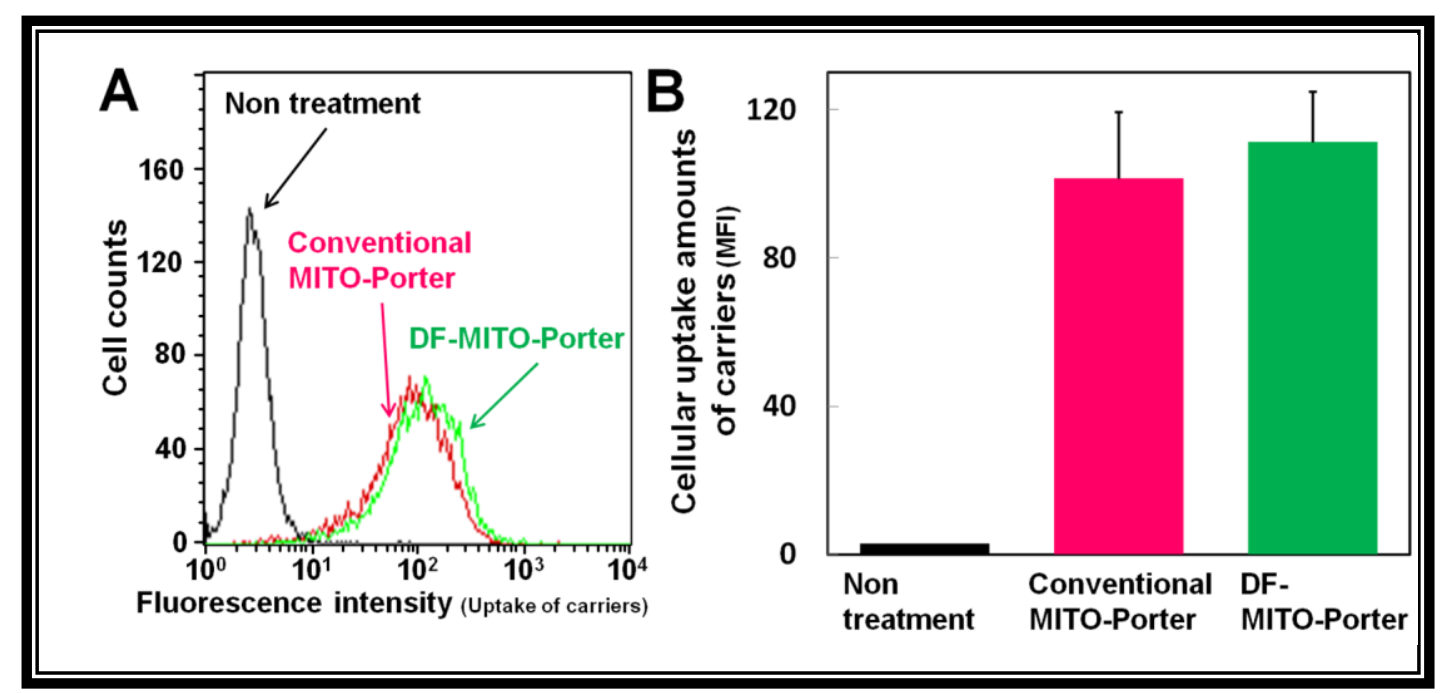

The histogram plot (A) shows the fluorescence intensities of the carriers labeled with NBD-

DOPE within cells after the transduction of the DF-MITO-Porter [green line] and the MITO-

Porter [pink line]. Black line indicates non-treated cells. The cellular uptake of the carrier (B).

Data are represented as the mean \pm S.D. $(n=3)$. 
3. Observation of cells after staining mitochondria with Rhodamine123 and MitoTracker Deep Red 633

We observed mitochondria stained with Rhodamine123 (green color) and MitoTracker Deep Red 633 (red pseudo color) to evaluate the mitochondrial membrane potential after the transduction of carriers. Merged images indicate yellow signals when mitochondria are stained with Rhodamine123 and MitoTracker Deep Red 633 are overlapped. Staining of mitochondria with Rhodamine 123 is dependent on the membrane potential, while MitoTracker Deep Red 633 stains mitochondria, even when membrane potential is lost. 
Figure S3. Images of mitochondria stained by Rhodamine123 and MitoTracker Deep Red 633

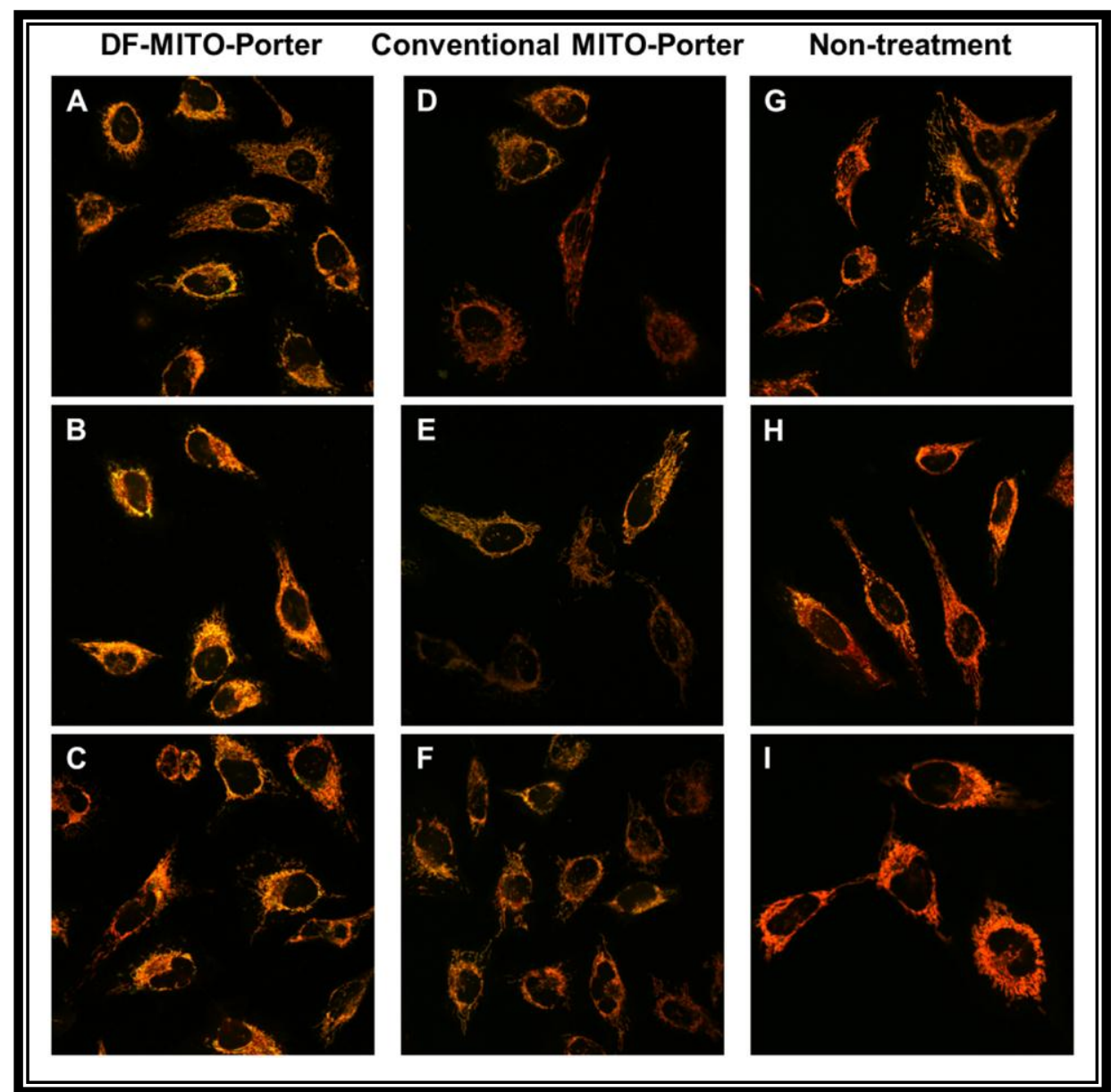

Merged images indicate yellow signals when mitochondria are stained with Rhodamine123 and MitoTracker Deep Red 633 are overlapped. A-C, DF-MITO-Porter; D-F, Conventional MITO-Porter: G-I, Non-treatment. 